\title{
Comment on; post-transcatheter aortic valve replacement acute kidney injury; prevention rather than cure
}

\author{
Macaulay Amechi Chukwukadibia Onuigbo ${ }^{1^{*}}$, Nneoma Agbasi ${ }^{2}$ \\ ${ }^{1}$ Department of Nephrology, Mayo Clinic Health System, Eau Claire, WI 54702, USA \\ ${ }^{2}$ North East London NHS Foundation Trust, UK
}

\section{AR T I C L E I N F O}

Article Type:

Letter to Editor

\section{Article History:}

Received: 28 August 2016

Accepted: 9 October 2016

Published online: 21 October 2016

\section{Keywords:}

Transcatheter aortic valve replacement, Surgical aortic valve replacement, Minimally invasive aortic valve replacement, Acute kidney injury, Renal replacement therapy, Hemodialysis

\begin{abstract}
Implication for health policy/practice/research/medical education:
There is newly accumulating evidence in the surgery-acute kidney injury (AKI) literature that intraoperative hypotension $(\mathrm{IOH})$ is a formidable and yet preventable causative factor in precipitating postoperative AKI. Postoperative AKI portends both increased short- and long-term morbidity and mortality, increased length of stay and higher healthcare costs. $\mathrm{IOH}$ is preventable. We present a case of AKI that led to the syndrome of rapid onset end stage renal disease (SORO-ESRD) in a 73-year-old diabetic hypertensive chronic kidney disease (CKD) male patient in 2012 following minimally invasive surgical aortic valve replacement. We call for more attention to be paid to $\mathrm{IOH}$ and to avoid too low blood pressures in the operating room. We posit that systolic blood pressure targets $\geq 90 \mathrm{~mm}$ $\mathrm{Hg}$ and/or MABP targets $\geq 60 \mathrm{~mm} \mathrm{Hg}$ potentially would improve postoperative renal outcomes.

Please cite this paper as: Onuigbo MAC, Agbasi N. Comment on; post-transcatheter aortic valve replacement acute kidney injury; prevention rather than cure. J Renal Inj Prev. 2017;6(2):12-15. DOI: 10.15171/jrip.2017.02
\end{abstract}

\section{Dear Editor,}

Huber et al in a single-center cohort study of 3646 patients who underwent inpatient vascular surgery between 2000 and 2010 at a tertiary care teaching hospital demonstrated that perioperative acute kidney injury (AKI) occurred in 1801 (49.4\%) patients (1). Besides, adjusted cardiovascular mortality estimates at 10 years were $17 \%, 31 \%, 30 \%$, and $41 \%$, for patients with no kidney disease, for patients with AKI but without chronic kidney disease (CKD), for patients with CKD but without $\mathrm{AKI}$, and for patients with $\mathrm{AKI}$ and CKD, respectively (1). Until very recently, in the cognate AKI-surgery literature, intraoperative hypotension $(\mathrm{IOH})$ following hypotensive anesthesia or controlled hypotension in the operating room has been accepted to not pose any significant short-term and/or long-term consequences on renal function (2). Nevertheless, in the last few years there has been an increasing awareness of the impact of $\mathrm{IOH}$ as a formidable albeit preventable factor in the causation of postoperative AKI (3-7).

AKI following aortic valve replacement (AVR) results in longer length of hospital stay, increased utilization of healthcare resources and at the same time is independently associated with both higher short- and long-term mortality $(8,9)$. AKI complicating surgical AVR (SAVR) has been variously reported in the literature (10-13). The increasing trend towards less invasive surgical options for the management of symptomatic AVR especially in patients with multiple comorbidities has led to such innovative surgical techniques as minimally invasive AVR, and recently, transcatheter AVR (TAVR) (14-16).

Very lately, Cheungpasitporn and colleagues from Mayo Clinic, Rochester, in an extensive review of the TAVR literature published in this journal, Journal of Renal Injury Prevention, noted that TAVR has now emerged as a viable treatment option for high-risk patients with severe aortic stenosis (AS) who are not suitable candidates for SAVR (17). The authors acknowledged that despite encouraging published outcomes, AKI after TAVR is common and lowers the survival of patients after TAVR (17). Factors identified to contribute to AKI following TAVR include 
preoperative factors (older age, pre-existing chronic kidney disease, iodinated contrast exposure, congestive heart failure, peripheral vascular disease, diabetes mellitus), periprocedural factors (bleeding and blood transfusion, embolic events, contrast agents, hypotension from rapid ventricular pacing, complicated cases requiring intraaortic balloon pump) and postoperative factors (vasoconstricting agents, nephrotoxins, decreased heart function, hemodynamic instability, grade of aortic regurgitation after the procedure) $(10-13,17)$.

From a preventative standpoint, a critical scrutiny of the above contributory factors to postoperative AKI following TAVR reveals that most of the factors with the exception of $\mathrm{IOH}$ and nephrotoxic exposure are non-modifiable factors $(6,7,18)$. IOH, albeit a very preventable causative factor, has for a long time been unrecognized and neglected as a very important contributing factor in precipitating postoperative AKI $(6,7)$. Moreover, postoperative AKI must be recognized in its full perspective - it must be conceded that AKI, including post-operative AKI, does sometimes lead to acute yet irreversible end stage renal disease, a syndrome of rapid onset end stage renal disease (SOROESRD), that we first described in the journal "Renal Failure," in 2010 (19).

Walsh et al analyzed 33330 non-cardiac surgeries at the Cleveland Clinic, Ohio and evaluated the association between intraoperative mean arterial pressure (MAP) from less than 55 to $75 \mathrm{~mm} \mathrm{Hg}$ and postoperative AKI and myocardial injury to determine the threshold of MAP where risk is increased (4). AKI and myocardial injury developed in $2478(7.4 \%)$ and $770(2.3 \%)$ surgeries, respectively. The MAP threshold where the risk for both outcomes increased was less than $55 \mathrm{~mm} \mathrm{Hg}$. Compared with never developing a MAP less than $55 \mathrm{~mm} \mathrm{Hg}$, those with a MAP less than $55 \mathrm{~mm} \mathrm{Hg}$ for 1-5, 6-10, 11-20, and more than 20 minutes had graded increases in their risk of the two outcomes (AKI: 1.18 [95\% CI, 1.06-1.31], 1.19 [1.03-1.39], 1.32 [1.11-1.56], and 1.51 [1.24-1.84], respectively; myocardial injury 1.30 [1.06-1.5], 1.47 [1.13-1.93], 1.79 [1.33-2.39], and 1.82 [1.31-2.55], respectively) (4). In a similar study, Sun et al, in a recent prospective cohort study of the association of IOH with AKI in 5127 patients undergoing non-cardiac surgery with invasive MAP monitoring and length of stay of one or more days in the hospital had established that AKI occurred in 324 (6.3\%) patients and was associated with MAP $<60 \mathrm{~mm} \mathrm{Hg}$ for 11-20 minutes, and MAP $<50 \mathrm{~mm} \mathrm{Hg}$ for more than 10 minutes in a clearly graded fashion (5).

\section{Case Report}

We present below a brief description of our experience in 2012 of a then 73-year old obese hypertensive diabetic CKD stage III Caucasian male patient seen at the Renal Unit of the Mayo Clinic Health System, Eau Claire, in Northwestern Wisconsin, USA, who developed acute yet irreversible AKI in March 2012 following a minimally invasive AVR for symptomatic AS and where IOH played a crucial instrumental role in precipitating AKI. The pa- tient, now 77 years old, remains on maintenance in-center outpatient hemodialysis, three times a week, for ESRD. We would argue that whereas if $\mathrm{IOH}$ was prevented here, the patient plausibly would have only experienced mild AKI, would not have needed renal replacement therapy (RRT) and would not be on maintenance hemodialysis four long years later, in March 2016.

A 73-year-old Caucasian man, nonsmoker with past medical history for hypertension, type II diabetes mellitus, obesity, sleep apnea, dyslipidemia, atrial fibrillation, osteoarthritis, a remote history of temporary need for hemodialysis in 2004 for AKI secondary to autoimmune hepatitis, with baseline serum creatinine of 1.5-1.90 mg/dL between November 2004 and February 2012 was evaluated for dyspnea in February 2012 (Figure 1). Outpatient medications included amlodipine $10 \mathrm{mg} / \mathrm{d}$, atenololchlorthalidone 100/25 daily, atorvastatin $80 \mathrm{mg} / \mathrm{d}$, insulin, lisinopril $40 \mathrm{mg} / \mathrm{d}$ and multivitamins with minerals daily. He was admitted to the CCU late in February 2012 with acutely worsening heart failure, severe dyspnea and chest pressure. Temperature 36.6, pulse $57 \mathrm{BPM}$, respiratory rate $35 / \mathrm{min}, \mathrm{BP} 147 / 76 \mathrm{~mm} \mathrm{Hg}$, and pulse oximetry $93 \%$ on 3 liters nasal cannula. HEENT; he had bilateral basal inspiratory crackles and a 3/6 ejection systolic ejection murmur predominantly in the aortic region. Hemoglobin was $10.7 \mathrm{~g} / \mathrm{dL}$ with otherwise normal CBC. Electrolytes were normal and serum creatinine was stable at $1.7 \mathrm{mg} / \mathrm{dL}$. BNP was 1314 pg/mL (0-100). Urinalysis was unremarkable, without dipstick proteinuria. His initial electrocardiogram (ECG) in the ED showed regular wide complex tachycardia of $120 \mathrm{BPM}$, in atrial flutter with left bundle branch block (LBBB). His chest x-ray showed pulmonary edema. The patient's chest pressure resolved following intravenous Furosemide and nitroglycerin $0.1 \mathrm{mcg} / \mathrm{kg} / \mathrm{min}$ infusion. He subsequently ruled out for acute Myocardial infarction (MI) by ECG and cardiac enzymes. Echocardiogram demonstrated a left ventricular ejection fraction (LVEF) of 50\%, paradoxical septal motion consistent with LBBB, grade 2/4 diastolic dysfunction with increased filling pressures, and severe AS with mean gradient of $48 \mathrm{~mm}$ $\mathrm{Hg}$, peak gradient of $73.6 \mathrm{~mm} \mathrm{Hg}$, peak velocity of $4.3 \mathrm{~m} / \mathrm{s}$, dimensionless index (LVOT/AV VTI ratio) of 0.25 , and

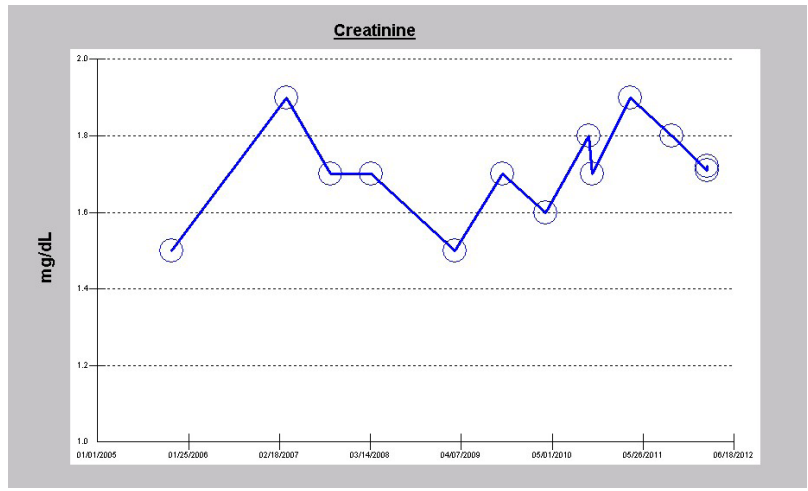

Figure 1. Stable serum creatinine of $1.5-1.9 \mathrm{mg} / \mathrm{dL}$ between November 2004 and February 2012. 
valve area of $1 \mathrm{~cm}^{2}\left(3-4 \mathrm{~cm}^{2}\right.$, normal).

He underwent coronary angiogram which did not show significant coronary artery disease. He underwent prophylactic dental extraction for severe caries and subsequently on March 2, 2012 a minimally invasive surgical AVR with a $25 \mathrm{~mm}$ St. Jude Epic stented tissue valve was performed with temporary pacing wires, right femoral venous percutaneous cannulation for cardiopulmonary bypass.

The AVR procedure was carried out through an $8 \mathrm{~cm}$ right anterior chest mid-third intercostal space incision, made directly over the fourth rib. One unit of packed red blood cells was given. The postoperative transesophageal echocardiogram demonstrated an LV of $50 \%$ with a normal functioning aortic valve prosthesis. The bypass time was 150 minutes. The cross clamp time was 101 minutes. Intraoperative continuous blood pressure recordings during the nearly 8 -hour long procedure demonstrated significant episodes of $\mathrm{IOH}$, with systolic blood pressure values as low as $50 \mathrm{~mm} \mathrm{Hg}$, MAP as low as $50 \mathrm{~mm} \mathrm{Hg}$, and diastolic blood pressure values as low as $44 \mathrm{~mm} \mathrm{Hg}$ repeatedly recorded during the operation (Figures 2 and 3). Because of post-operative AKI, progressive volume overload and oligoanuria despite high-dose continuous intravenous furosemide infusion, he needed RRT to be initiated within 24 hours of the AVR. He had been on chronic ACE inhibition for many years with Lisinopril $40 \mathrm{mg}$

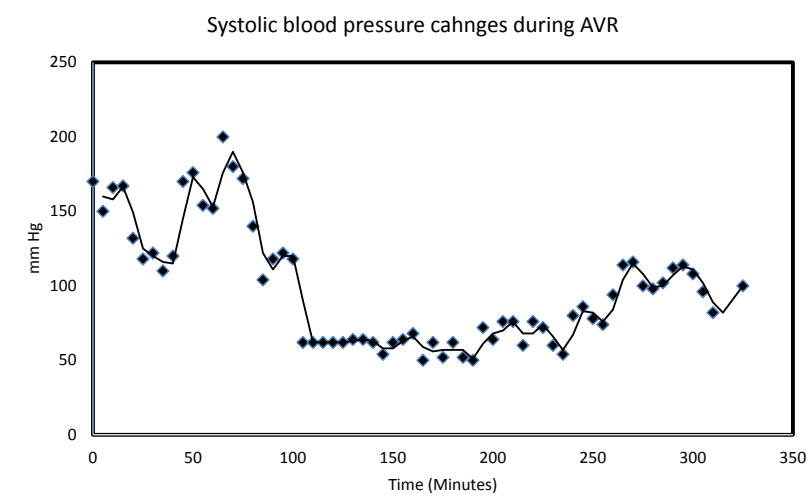

Figure 2. Systolic blood pressure trajectory during the minimally invasive AVR procedure in early March 2012.

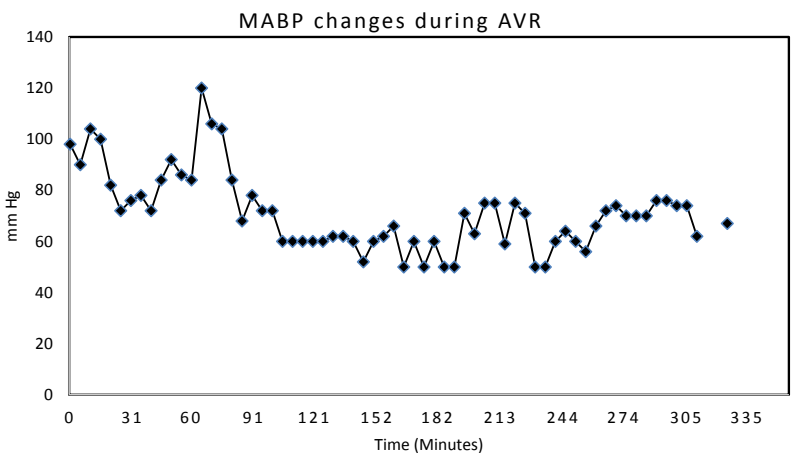

Figure 3. Mean arterial blood pressure trajectory during the minimally invasive AVR procedure in early March 2012. daily immediately prior to the AVR procedure; last dose of Lisinopril documented was on February 26, 2012 as an inpatient. Serum creatinine rose very quickly and has since remained elevated (Figures 4 and 5). His initial hemodialysis vascular access was a temporary right femoral vein dialysis catheter, on March 3, 2012. The right femoral dialysis catheter was subsequently replaced by a left internal jugular vein tunneled Palindrome dialysis catheter (Permcath) for dialysis access on March 15, 2012. Four months later, when it became clear that there was no renal recovery, his status was converted to ESRD, and a transposed cephalic vein AV fistula was created in the left upper arm on July 10, 2012. This was converted in January 2013 to a left upper arm loop graft die to poor maturation of the prior placed AVF. He has since then continued his hemodialysis treatments using this AVF graft to date. He remains an anuric ESRD patient, with a serum creatinine of $8.85 \mathrm{mg} / \mathrm{dL}$ as at March 2, 2016, exactly four years after the minimally invasive AVR procedure (Figure 5).

\section{Conclusion}

Increasingly, there is accumulating evidence that $\mathrm{IOH}$ is a formidable and yet preventable causative factor in precipitating postoperative AKI. Postoperative AKI portends both increased short- and long-term mortality. AKI can

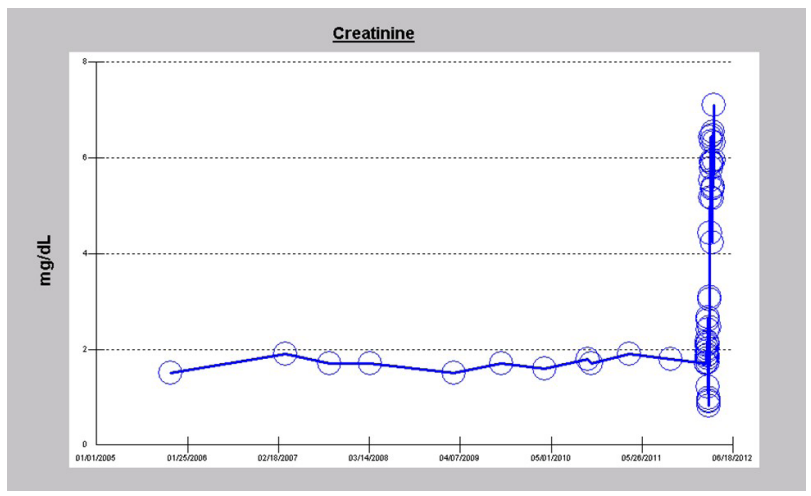

Figure 4. Serum creatinine trajectory soon following the minimally invasive AVR procedure in early March 2012.

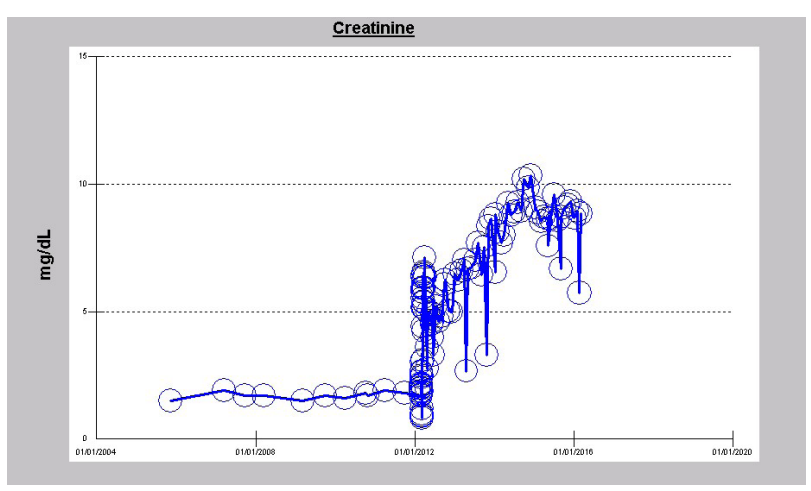

Figure 5. Serum creatinine trajectory from November 2004, immediately following the minimally invasive AVR procedure in early March 2012, through early March 2016 
indeed sometimes lead to acute yet irreversible and permanent renal failure, the syndrome of rapid onset end stage renal disease (SORO-ESRD). IOH is preventable. We strongly posit that every effort to eliminate $\mathrm{IOH}$ in the operating room is worth every effort $(6,7,20)$. Randomized controlled trials to test for the most appropriate level of intraoperative blood pressures to prevent the precipitation of postoperative AKI are long overdue. Furthermore, from our anecdotal experiences here at the Mayo Clinic Health System in Northwestern Wisconsin, elective surgical procedures, both cardiac and non-cardiac, should be deferred until hypertension is controlled $(6,7,20)$. As a result, the consequences of $\mathrm{IOH}$ on renal outcomes will be mitigated or at least minimized $(6,7,20)$.

\section{Authors' contribution}

MACO; conception, design, acquisition of data, data analysis, interpretation of data, literature review, drafting the article and final approval of manuscript. NA; literature review, drafting the article and final approval of manuscript.

\section{Conflicts of interest}

None.

\section{Ethical considerations}

Ethical issues (including plagiarism, data fabrication, double publication) have been completely observed by the authors. Written consent was obtained from the patient for publication of the study.

\section{Funding/Support}

None.

\section{References}

1. Huber M, Ozrazgat-Baslanti T, Thottakkara P, Scali S, Bihorac A, Hobson C. Cardiovascular-specific mortality and kidney disease in patients undergoing vascular surgery. JAMA Surg. 2016;151:441-50. doi: 10.1001/ jamasurg.2015.4526.

2. Choi WS, Samman N. Risks and benefits of deliberate hypotension in anaesthesia: A systematic review. Int J Oral Maxillofac Surg. 2008;37:687-703. doi: 10.1016/j. ijom.2008.03.011.

3. Brady K, Hogue CW. Intraoperative hypotension and patient outcome: does "one size fit all?" Anesthesiology. 2013;119:495-7. doi: 10.1097/ALN.0b013e3182a10cce.

4. Walsh M, Devereaux PJ, Garg AX, Kurz A, Turan A, Rodseth $\mathrm{RN}$, et al. Relationship between intraoperative mean arterial pressure and clinical outcomes after noncardiac surgery: toward an empirical definition of hypotension. Anesthesiology. 2013;119:507-15. doi: 10.1097/ALN.0b013e3182a10e26.

5. Sun LY, Wijeysundera DN, Tait GA, Beattie WS. Association of intraoperative hypotension with acute kidney injury after elective noncardiac surgery. Anesthesiology. 2015;123:51523. doi: 10.1097/ALN.0000000000000765.
6. Onuigbo MA, Agbasi N. Intraoperative hypotension - a neglected causative factor in hospital-acquired acute kidney injury; a Mayo Clinic Health System experience revisited. J Renal Inj Prev. 2015;4:61-7. doi: 10.12861/jrip.2015.13.

7. Onuigbo MA, Agbasi N. Association of intraoperative hypotension with acute kidney injury after elective noncardiac surgery-prevention is better than cure. Ren Fail. 2016;4:1-2. doi: 10.3109/0886022X.2015.1128448.

8. Chertow GM, Burdick E, Honour M, Bonventre JV, Bates DW. Acute kidney injury, mortality, length of stay, and costs in hospitalized patients. J Am Soc Nephrol. 2005;16:336570. doi: 10.1681/ASN.2004090740.

9. Ricci Z, Cruz D, Ronco C. The RIFLE criteria and mortality in acute kidney injury: a systematic review. Kidney Int. 2008;73:538-46.

10. Thongprayoon C, Cheungpasitporn W, Srivali N, Ungprasert P, Kittanamongkolchai W, Greason KL, et al. Acute kidney injury after transcatheter aortic valve replacement: a systematic review and meta-analysis. Am J Nephrol. 2015;41:372-82. doi: 10.1159/000431337.

11. Elhmidi Y, Bleiziffer S, Deutsch MA, Krane M, Mazzitelli $\mathrm{D}$, Lange R, et al. Acute kidney injury after transcatheter aortic valve implantation: incidence, predictors and impact on mortality. Arch Cardiovasc Dis. 2014;107:133-9. doi: 10.1016/j.acvd.2014.01.002.

12. Scherner $M$, Wahlers $T$. Acute kidney injury after transcatheter aortic valve implantation. J Thorac Dis. 2015;7:1527-35.

13. Najjar M, Salna M, George I. Acute kidney injury after aortic valve replacement: Incidence, risk factors and outcomes. Expert Rev Cardiovasc Ther. 2015;13:301-16.

14. O'Brien SM, Shahian DM, Filardo G, Ferraris VA, Haan CK, et al; Society of Thoracic Surgeons Quality Measurement Task Force. The Society of Thoracic Surgeons 2008 cardiac surgery risk models: part 2--isolated valve surgery. Ann Thorac Surg. 2009;88:S23-42. doi: 10.1016/j. athoracsur.2009.05.056.

15. Adams DH, Popma JJ, Reardon MJ, Yakubov SJ, Coselli JS, Deeb GM, et al. Transcatheter aortic-valve replacement with a self-expanding prosthesis. N Engl J Med. 2014;370:17908. doi: 10.1056/NEJMoa1400590.

16. Thongprayoon C, Cheungpasitporn W, Srivali N, Harrison AM, Gunderson TM, Kittanamongkolchai W, et al. AKI after transcatheter or surgical aortic valve replacement. J Am Soc Nephrol. 2016;27:1854-60. doi: 10.1681/ASN.2015050577.

17. Cheungpasitporn W, Thongprayoon C, Kashani K. Transcatheter aortic valve replacement: a kidney's perspective. J Renal Inj Prev. 2016;5:1-7. doi: 10.15171/ jrip.2016.01.

18. Yamout H, Levin ML, Rosa RM, Myrie K, Westergaard S. Physician prevention of acute kidney injury. Am J Med. 2015;128:1001-6. doi: 10.1016/j.amjmed.2015.04.017.

19. Onuigbo MA. Syndrome of rapid-onset end-stage renal disease: a new unrecognized pattern of CKD progression to ESRD. Ren Fail. 2010;32:954-8. doi: $10.3109 / 0886022$ X.2010.502608.

20. Onuigbo MA. Perioperative acute kidney injury: prevention rather than cure. JAMA Surg. 2016;151:782-3.

Copyright (c) 2017 The Author(s); Published by Nickan Research Institute. This is an open-access article distributed under the terms of the Creative Commons Attribution License (http://creativecommons.org/licenses/by/4.0), which permits unrestricted use, distribution, and reproduction in any medium, provided the original work is properly cited. 